\title{
Advanced Gauss Pseudospectral Method for Continuous-time Hammerstein System Identification
}

\author{
HE Ying,DAI Ming-xiang,YANG Xin-min,YI Wen-jun
}

\author{
Science and Technology on Transient Physics Laboratory, Nanjing University of Science and \\ Technology, Nanjing 210094 P.R. China
}

heying2172@foxmail.com,916047597@qq.com,yxm@mail.njust.edu.cn,yiwenjun0@163.com

\begin{abstract}
Keywords: Advanced Gauss Pseudospectral Method; Continuous-time Hammerstein System; Parameter Identification; Nonlinear Programming Problem
\end{abstract}

\begin{abstract}
In the paper, an advanced Gauss pseudospectral method (AGPM) is proposed to estimate the parameters of the continuous-time (CT) Hammerstein model consisting of a CT linear block followed by a static nonlinearity. The basic idea of AGPM is to transcribe the CT identification problem into a discrete nonlinear programming problem (NLP), which can be solved with the well-developed sequential quadratic programming (SQP) algorithm. The nonlinear part of the Hammerstein system is approximated with the Gauss pseudospectral approximation method. The linear part is written as a controllable canonical form. AGPM can converge to the true values of the CT Hammerstein model with few interpolated Legendre-Gauss (LG) nodes. Lastly illustrative examples are proposed to verify the accuracy and efficiency of the method.
\end{abstract}

\section{Introduction}

The identification of the block-oriented nonlinear systems has been an active research area for the last several decades. A block-oriented nonlinear system represents a kind of nonlinear dynamical system consisting of a linear dynamic system and a static nonlinear block, mainly including Hammerstein ${ }^{[1,2]}$, Wiener ${ }^{[3]}$, Hammerstein-Wiener ${ }^{[4]}$ and Wiener-Hammerstein systems ${ }^{[5]}$. A typical Hammerstein system is composed of a static nonlinear block followed by a dynamic linear block ${ }^{[6]}$. It is significant to make the identification of Hammerstein system due to the wide application in industrial engineering, signal processing, power system and et al. There have been many methods proposed in decades for parameters identification of Hammerstein system, such as over-parameterization method, stochastic method ${ }^{[7]}$, separable least squares method ${ }^{[\underline{[}]}$, blind method [9], frequency domain method ${ }^{[10]}$, subspace $\operatorname{method~}^{[11]}$ and iterative method ${ }^{[7]}$. However, most existing research about the parameters identification of Hammerstein model are focused on the discrete-time (DT) case, regardless of the fact that it is easier to capture the plant dynamic intuitively in CT rather than DT. Only a small number of researches are relative to the CT Hammerstein model, such as W. Greblicki ${ }^{[12,13]}$ proposed a non-parametric method for idenfication of CT Hammerstein model using a kernel algorithm; Ximing ${ }^{[14]}$ solved the same problems with multiple step responses on the CT Hammerstein system.

AGPM [15] trancribes the parameters identificaton of the CT Hammerstein system into the corresponding NLP. The CT Hammerstein system is descretized with LG nodes, which is nonuniform with a higher density at the end of the time interval. The most important advantages $^{[15,16]}$ of AGPM are the high precision and rapid convergence on the CT dynamic system. AGPM was first proposed by Benson in Ref.[16] and widely applied for solving optimal control problems and trajectory optimization. Additionally, Gauss pseudospectral approximation can also be used to approximation the static nonlinearity in the CT Hammerstein system and then rewrite the Hammerstein as a multiple input and single output linear (MISO) system.

The contributions of this paper are as follows. The advanced Gauss pseudospectral method is proposed for the parameter identification of the CT Hammerstein system. AGPM can directly transform the CT problems into the discrete-time NLP with a small number of points (less than 50). The nonlinear part of the CT Hammerstein system is approximated with Lagrangian polynomials, 
and the CT linear part is expressed as state-space form instead of transfer function form to circumvent the high order time-derivative of the I/O data. The estimated parameters with AGPM converge to the true values quickly. It is significant to figure out that AGPM can identify all the parameters with one excitement. Furthermore, AGPM has been illustrated with one typical numerical examples to show the good performance of identifying the parameters of the CT Hammerstein system.

\section{Advanced Gauss Pseudospectral Method}

Pseudospectral approximation is a kind of linear combination with the Lagrange basis functions, and it is based on the theory of orthogonal collocation where the collocation nodes are chosen as Legendre-Gauss (LG) points. The LG collocation points are obtained by the roots of Legendre polynomial defined on the time domain of $(-1,1)$. Most significantly, The LG points allocate non-uniformly through the time domain and higher density of nodes near the endpoints, as which the Runge phenomenon is effectively restrained.

The location of the nodes in the AGPM are based on Legendre-Gauss (LG) points ${ }^{[16]}$, which lie in the interval $(-1,1)$. LG points include neither the initial point nor the final point, denoting as $\tau_{1}, \tau_{2}, \cdots, \tau_{N}$, where $-1<\tau_{1}<\tau_{2}<\cdots<\tau_{N}<1$. In order to satisfy with the endpoint constraints, an initial-time point $\tau_{0}=-1$ and a final-time point $\tau_{N+1}=1$ are defined. The state and control variables in AGPM are approximated with the different Lagrange interpolation polynomials. The state is approximated with a set of $N+1$ Lagrange interpolating polynomials

$$
\boldsymbol{x}(\tau) \approx \boldsymbol{x}^{N}(\tau)=\sum_{l=0}^{N} X_{l} \phi_{l}(\tau)
$$

Where $X_{l}=\boldsymbol{x}\left(\tau_{l}\right),(l=0,1, \cdots, N)$ and $\phi_{l}(\tau),(l=0,1, \cdots, N)$ are defined as

$$
\phi_{l}(\tau)=\prod_{j=0, j \neq l}^{N} \frac{\tau-\tau_{j}}{\tau_{l}-\tau_{j}} \quad(l=0,1, \cdots, N) .
$$

While the control is approximated with a set of $N$ Lagrange interpolation polynomials, described as

$$
\boldsymbol{u}(\tau) \approx \boldsymbol{u}^{N}(\tau)=\sum_{l=1}^{N} U_{l} \phi_{l}^{*}(\tau)
$$

Where $U_{l}=\boldsymbol{u}\left(t_{l}\right),(l=1, \cdots, N)$ and $\phi_{l}^{*}(\tau),(l=1, \cdots, N)$ are defined as

$$
\phi_{l}^{*}(\tau):=\prod_{j=1, j \neq l}^{N} \frac{\tau-\tau_{j}}{\tau_{l}-\tau_{j}} \quad(l=1, \cdots, N) .
$$

It is noted that the interpolation polynomials of the state variables is composed of the initial-time point $\tau_{0}$ and LG points, while the interpolation polynomials of the control variables just using LG points. According to(3), AGPM does not contain control values at the initial-time. Additionally, it is obviously seen that both equations (2) and (4) satisfy with the $\delta$-function property as

$$
\begin{aligned}
& \phi_{l}\left(\tau_{j}\right)= \begin{cases}1 & \text { if } l=j \\
0 & \text { if } l \neq j,\end{cases} \\
& \phi_{l}^{*}\left(\tau_{j}\right)= \begin{cases}1 & \text { if } l=j \\
0 & \text { if } l \neq j .\end{cases}
\end{aligned}
$$

Under the $\delta$-function properties of (5) and (6), the approximated state and control variables are equal to the true values at the LG time points, displaying as

$$
\begin{aligned}
& \boldsymbol{x}\left(\tau_{i}\right)=\boldsymbol{x}^{N}\left(\tau_{i}\right), i=0,1, \cdots, N, \\
& \boldsymbol{u}\left(\tau_{i}\right)=\boldsymbol{u}^{N}\left(\tau_{i}\right), i=1,2, \cdots, N .
\end{aligned}
$$

Differentiating the expression of (1) and solving the function value at LG points, then we derive

$$
\dot{\boldsymbol{x}}\left(\tau_{k}\right) \approx \dot{\boldsymbol{x}}^{N}\left(\tau_{k}\right)=\sum_{l=0}^{N} X_{l} \dot{\phi}_{l}\left(\tau_{k}\right)=\sum_{l=0}^{N} D_{k l} X_{l} \quad k=1, \cdots, N .
$$

where 


$$
D_{k l}=\dot{\phi}_{l}\left(\tau_{k}\right)=\sum_{i=0, i \neq l}^{N} \frac{\prod_{j=0, j \neq l, i}^{N}\left(t_{k}-t_{j}\right)}{\prod_{j=0, j \neq l}^{N}\left(t_{l}-t_{j}\right)}, k=1, \cdots, N ; L=0,1, \cdots, N .
$$

Define a new matrix $\boldsymbol{D}$ composed of $D_{k l}, k=1, \cdots, N, L=0,1, \cdots, N$, named as Gauss pseudospectral differential matrix. The integration part of the cost function is approximated by Gaussian quadrature method ${ }^{[16]}$. Define the Gauss weights with $\omega_{k}, k=1, \cdots, N$, and then the integration in the cost functional can be further rewritten as

$$
\int_{-1}^{1} g\left(\boldsymbol{x}(\tau), \boldsymbol{u}(\tau), \tau ; t_{0}, t_{f}\right) \mathrm{d} \tau=\sum_{k=1}^{N} \omega_{k} g\left(X_{k}, U_{k}, \tau_{k} ; t_{0}, t_{f}\right),
$$

Note that the derivative of state is collocated only at the LG points and not at the boundary points. In order to compensate this drawback, the connection of the initial-time state and final-time state is built as

$$
\boldsymbol{x}\left(\tau_{N+1}\right)-\boldsymbol{x}\left(\tau_{0}\right)=\int_{-1}^{1} \dot{\boldsymbol{x}}(\tau) \mathrm{d} \tau=\sum_{k=1}^{N} \omega_{k} \dot{\boldsymbol{x}}\left(\tau_{k}\right) .
$$

Replace $\dot{\boldsymbol{x}}\left(\tau_{k}\right), k=1,2, \cdots, N$ with (9) to derive

$$
X_{N+1}=X_{0}+\sum_{k=1}^{N} \sum_{l=0}^{N} \omega_{k} D_{k l} X_{l},
$$

where $X_{N+1}=\boldsymbol{x}\left(\tau_{N+1}\right)$ represents the final-time value of state. Equation (13) uses $D_{k l} X_{l}$ to replace the dynamic formula $f\left(\boldsymbol{x}\left(\tau_{k}\right), \boldsymbol{u}\left(\tau_{k}\right), \tau_{k} ; t_{0}, t_{f}\right)$ in Ref.[16] to linearize the extra endpoint constraint on the state. Such replacement is called as the advanced GPM, which can decrease the computation burden of the nonlinear programming (NLP) problem.

To discretize the CT optimal problem in (13)-(17) with AGPM, apply the discretization of (11) to the Lagrangian part of (13), the approximation of (9) to the left side of (14) and combine the extra final-time state constraint of (13), then the discretized form of the Bolza CT optimal problem is rewritten into a NLP problem as below.

Minimize the cost functional

$$
J=\frac{t_{f}-t_{0}}{2} \sum_{k=1}^{N} \omega_{k} g\left(X_{k}, U_{k}, \tau_{k} ; t_{0}, t_{f}\right),
$$

subject to

$$
\begin{gathered}
\sum_{l=0}^{N} D_{k l} X_{l}=\frac{t_{f}-t_{0}}{2} f\left(X_{k}, U_{k}, \tau_{k} ; t_{0}, t_{f}\right), \quad k=1, \cdots, N, \\
\psi\left(X_{0}, X_{N+1} ; t_{0}, t_{f}\right)=\mathbf{0}, \\
X_{N+1}=X_{0}+\sum_{k=1}^{N} \sum_{l=0}^{N} \omega_{k} D_{k l} X_{l} . \\
x_{i}^{L} \leq x_{i}\left(\tau_{k}\right) \leq x_{i}^{U}, i=1,2, \cdots, n ; k=1,2, \cdots, N, \\
u_{i}^{L} \leq u_{i}\left(\tau_{k}\right) \leq u_{i}^{U}, i=1,2, \cdots, m ; k=1,2, \cdots, N .
\end{gathered}
$$

\section{The CT Hammerstein system}

\section{The CT Hammerstein system model}

The CT Hammerstein system is composed of a CT dynamic linear system and a static nonlinear system, and the linear part is followed by the nonlinearity. The output $y_{u}(t)$ is contaminated by the zero-mean white noise $v(t)$ shown in Fig.1

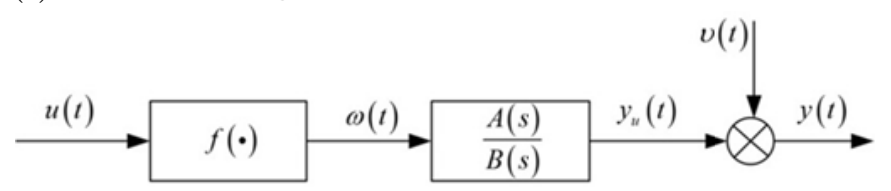

Fig.1 The CT Hammerstein system block diagram

The CT Hammerstein system can be mathematically written as below ${ }^{[14]}$, 


$$
\left\{\begin{array}{l}
\omega(t)=f(u(t)) \\
G(s)=\frac{Y_{u}(s)}{\Omega(s)}=\frac{A(s)}{B(s)}=\frac{b_{m} s^{m}+b_{m-1} s^{m-1}+\cdots+b_{1} s+b_{0}}{a_{n} s^{n}+a_{n-1} s^{n-1}+\cdots+a_{1} s+1} \\
y(t)=y_{u}(t)+v(t),
\end{array}\right.
$$

where

$$
\begin{aligned}
& a_{i} \in \square \quad i=1,2, \cdots, n, \\
& b_{k} \in \square \quad k=1,2, \cdots, m \quad m<n .
\end{aligned}
$$

The nonlinear function $f(\cdot)$ and parameters $a_{i}(i=1,2, \cdots, n), b_{k}(k=1,2, \cdots, m)$ are all unknown and need to be identified. The dynamic linear subsystem $G(s)$ is assumed to be a minimum phase system. If we make the first part of CT Hammerstein system multiplied by $k_{g}\left(k_{g} \neq\right.$ 0 ) and the linear part by $1 / k_{g}$, the whole system can keep same with the previous one. The equivalent system can be shown in Fig. 2.

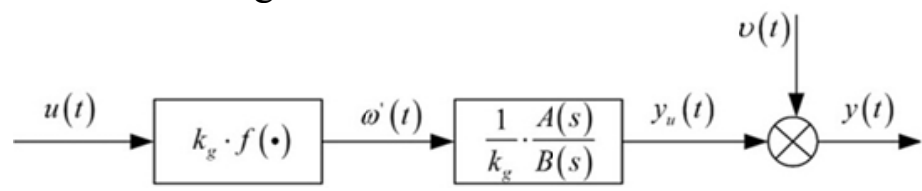

Fig. 2 The equivalent CT Hammerstein system block diagram

$$
\left\{\begin{array}{l}
\omega^{\prime}(t)=k_{g} f(u(t)) \\
G^{\prime}(s)=\frac{Y_{u}(s)}{\Omega^{\prime}(s)}=\frac{1}{k_{g}} \frac{b_{m} s^{m}+b_{m-1} s^{m-1}+\cdots+b_{1} s+b_{0}}{a_{n} s^{n}+a_{n-1} s^{n-1}+\cdots+a_{1} s+1}
\end{array}\right.
$$

Consequently, the parameters in the nonlinearity and the linear transfer function are varied with the arbitrary non-zero gain $k_{g}$. It is impossible to make the parameters estimation when the parameters in the system is not unique. In order to circumvent the ambiguity, a uniqueness assumption is proposed here by setting up $k_{g}=b_{0}$. As a result, the CT Hammerstein system becomes,

$$
\left\{\begin{array}{l}
\omega(t)=f(u(t)) \\
G(s)=\frac{Y_{u}(s)}{\Omega(s)}=\frac{A(s)}{B(s)}=\frac{b_{m} s^{m}+b_{m-1} s^{m-1}+\cdots+b_{1} s+1}{a_{n} s^{n}+a_{n-1} s^{n-1}+\cdots+a_{1} s+1} \\
y(t)=y_{u}(t)+v(t) .
\end{array}\right.
$$

Actually the parameters $\left(b_{m}, \cdots, b_{1}\right)$ in (22) differ from the corresponding parameters in (21). But to be consistent with previous publications, we still choose $\left(b_{m}, \cdots, b_{1}\right)$ as the parameters notations in the numerator of linear part.

In the linear part of the CT Hammerstein system shown in (22), the system order is $n$, which means that the largest order of time derivative of state variable is $n$. The state-space form for the linear part of the CT Hammerstein system is shown as

$$
\left\{\begin{array}{l}
\dot{\boldsymbol{x}}(t)=\boldsymbol{A} \boldsymbol{x}(t)+\boldsymbol{B} \omega(t) \\
\boldsymbol{y}(t)=\boldsymbol{C} \boldsymbol{x}(t)+v(t),
\end{array}\right.
$$

where $\boldsymbol{x}(t) \in \mathbb{R}^{n}$ is the state vector; $\boldsymbol{A} \in \mathbb{R}^{n \times n}$ is the system matrix, $\boldsymbol{B} \in \mathbb{R}^{n \times 1}$ is the input matrix; $\boldsymbol{C} \in \mathbb{R}^{1 \times n}$ is the output matrix. they are all respectively defined as

$$
\begin{gathered}
\boldsymbol{x}(t)=\left[\begin{array}{cccc}
x_{1}(t) & x_{2}(t) & \cdots & x_{n}(t)
\end{array}\right]^{T}, \\
\boldsymbol{A}=\left[\begin{array}{ccccc}
0 & 1 & 0 & \cdots & 0 \\
0 & 0 & 1 & \cdots & 0 \\
\vdots & \vdots & \vdots & \ddots & 0 \\
-\frac{1}{a_{n}} & -\frac{a_{1}}{a_{n}} & -\frac{a_{2}}{a_{n}} & \cdots & -\frac{a_{n-1}}{a_{n}}
\end{array}\right], \\
\boldsymbol{B}=\left[\begin{array}{llllll}
0 & 0 & 0 & \cdots & 1
\end{array}\right]^{T},
\end{gathered}
$$




$$
\boldsymbol{C}=\left[\begin{array}{lllllll}
\frac{1}{a_{n}} & \frac{b_{1}}{a_{n}} & \cdots & \frac{b_{m}}{a_{n}} & 0 & \cdots & 0
\end{array}\right] .
$$

\section{The CT optimal control problem description}

The Bolza CT optimal problem with initial time $t_{0}$, and final time $t_{f}$, can be generalized as followings.

Minimize the cost functional

$$
J=\Phi\left(\boldsymbol{x}\left(t_{0}\right), \boldsymbol{x}\left(t_{f}\right) ; t_{0}, t_{f}\right)+\int_{t_{0}}^{t_{f}} g\left(\boldsymbol{x}(t), \boldsymbol{u}(t), t ; t_{0}, t_{f}\right) \mathrm{d} t,
$$

subject to

$$
\begin{gathered}
\frac{\mathrm{d} \boldsymbol{x}(t)}{\mathrm{d} t}=f\left(\boldsymbol{x}(t), \boldsymbol{u}(t), t ; t_{0}, t_{f}\right) \in \square^{n}, \\
\boldsymbol{\psi}\left(\boldsymbol{x}\left(t_{0}\right), \boldsymbol{x}\left(t_{f}\right) ; t_{0}, t_{f}\right)=\mathbf{0} \in \square^{q}, \\
x_{i}^{L} \leq x_{i}(t) \leq x_{i}^{U}, \mathrm{i}=1,2, \cdots, n, \\
u_{i}^{L} \leq u_{i}(t) \leq u_{i}^{U}, \mathrm{i}=1,2, \cdots, m,
\end{gathered}
$$

where $\boldsymbol{u}(t) \in \mathbb{R}^{m}$ and $\boldsymbol{x}(t) \in \mathbb{R}^{n}$ are row vectors to represent the control variables and state variables, $x_{i}(t), i=1,2, \cdots, n$ are the elements of $\boldsymbol{x}(t)$ and $u_{i}(t), i=1,2, \cdots, m$ are the elements of $\boldsymbol{u}(t) ; \Phi: \mathbb{R}^{n} \times \mathbb{R}^{m} \times \mathbb{R} \times \mathbb{R} \rightarrow \mathbb{R}$ denotes the Mayer part of the cost function, $g: \mathbb{R}^{n} \times \mathbb{R}^{m} \times$ $\mathbb{R} \times \mathbb{R} \times \mathbb{R} \rightarrow \mathbb{R}$ is the Lagrangian part; $g: \mathbb{R}^{n} \times \mathbb{R}^{m} \times \mathbb{R} \times \mathbb{R} \times \mathbb{R} \rightarrow \mathbb{R}^{n}$ denotes the system dynamic equation; $\boldsymbol{\Psi}: \mathbb{R}^{n} \times \mathbb{R}^{n} \times \mathbb{R} \times \mathbb{R} \rightarrow \mathbb{R}^{q}$ denotes the boundary constraint; $x_{i}^{L}, x_{i}^{U}, i=$ $1,2, \cdots, n$ are the lower bounds and upper bounds of the state variables and $u_{i}^{L}, u_{i}^{U}, i=1,2, \cdots, m$ are the lower bounds and upper bounds of the control variables.

In Bolza optimal control problem, the time scale is located in $\left[t_{0}, t_{f}\right]$, but the GPM is based on the time interval $[-1,1]$.A bilinear transformation ${ }^{[18]}$ from the time interval $t \in\left[t_{0}, t_{f}\right]$ into $\tau \in[-1,1]$ is widely used by researchers as

$$
t=\frac{t_{f}-t_{0}}{2} \tau+\frac{t_{f}+t_{0}}{2} .
$$

Then make a time derivative of (33)

$$
\mathrm{d} t=\frac{t_{f}-t_{0}}{2} \mathrm{~d} \tau
$$

Therefore, the Bolza optimal control problem (28)-(32) can be descripted as the dependent variable $\tau$ with the time domain $[-1,1]$, showing as below.

Minimize the cost functional

$$
J=\Phi(x(+1), x(-1) ;-1,+1)+\frac{t_{f}-t_{0}}{2} \int_{-1}^{1} g\left(x(\tau), \boldsymbol{u}(\tau), \tau ; t_{0}, t_{f}\right) \mathrm{d} \tau,
$$

subject to

$$
\begin{gathered}
\frac{\mathrm{d} \boldsymbol{x}}{\mathrm{d} \tau}=\frac{t_{f}-t_{0}}{2} f\left(\boldsymbol{x}(\tau), \boldsymbol{u}(\tau), \tau ; t_{0}, t_{f}\right), \\
\psi\left(x(-1), x(1) ; t_{0}, t_{f}\right)=\mathbf{0} \in \square^{q}, \\
x_{i}^{L} \leq x_{i}(\tau) \leq x_{i}^{U}, i=1,2, \cdots, n, \\
u_{i}^{L} \leq u_{i}(\tau) \leq u_{i}^{U}, \mathrm{i}=1,2, \cdots, m .
\end{gathered}
$$

\section{Parameters Identification of CT Hammerstein System}

\section{State-space form transformation of hammerstein system}

As we all known, the higher the order of time derivative, the larger the amplification of the estimation noise. Therefore, the transformation of the linear part from transfer function form into state-space form can prompt the accuracy of the CT Hammerstein system identification, as the order of time derivative in state-space form is only equal to one. In addition, the state-space form is fit for the application of AGPM. 
The static nonlinear function, which is the first part of CT Hammerstein system, can be approximated by a linear combination of finite set of basis functions as $f(u)=\sum_{i=1}^{p} c_{i} \psi_{i}(u)$, where $c_{i}$ are coefficients which need to be estimated and $\psi_{i}(\cdot)$ are user chosen functions, $p$ is equal to the number of the basis functions, and define a new variable $\theta \in \mathbb{R}^{(n+m+k) \times 1}$ as

$$
\theta=\left[\begin{array}{llllllllll}
\frac{1}{a_{n}} & \frac{a_{1}}{a_{n}} & \cdots & \frac{a_{n-1}}{a_{n}} & \frac{b_{1}}{a_{n}} & \cdots & \frac{b_{m}}{a_{n}} & c_{1} & \cdots & c_{K}
\end{array}\right]^{T},
$$

Then the state equation (23) can be further rewritten as

$$
\left\{\begin{aligned}
\dot{x}_{1}(t)= & x_{2}(t), \\
\dot{x}_{2}(t)= & x_{3}(t), \\
& \vdots \\
\dot{x}_{n}(t)= & -\theta_{1} x_{1}(t)-\theta_{2} x_{2}(t) \cdots-\theta_{n} x_{n}(t)+\sum_{i=1}^{K} \theta_{n+m+i} \phi_{i}\left(u_{\tau}\right), \\
& y(t)=\theta_{1} x_{1}(t)+\theta_{n+1} x_{2}(t) \cdots+\theta_{n+m} x_{m+1}(t) .
\end{aligned}\right.
$$

From the view of mathematics, we can take account into the system identification problem as an optimal problem to minimize the output error of system, then the estimated parameters are respectively became the optimal variables of the optimal problem. Define the new variables with a hat as the estimation of the system variables in (41)-(42). Therefore, the corresponding optimal problem of the CT Hammerstein system identification is showing as below.

Minimize the cost functional

$$
J=\int_{t_{0}}^{t_{f}}(y(t)-\hat{y}(t))^{2} \mathrm{~d} t
$$

subject to

$$
\left\{\begin{aligned}
\dot{\hat{x}}_{1}(t) & =\hat{x}_{2}(t) \\
\dot{\hat{x}}_{2}(t) & =\hat{x}_{3}(t) \\
& \vdots \\
\dot{\hat{x}}_{n}(t)= & -\hat{\theta}_{1} \hat{x}_{1}(t)-\hat{\theta}_{2} \hat{x}_{2}(t) \cdots-\hat{\theta}_{n} \hat{x}_{n}(t)+\sum_{i=1}^{K} \hat{\theta}_{n+m+i} \phi_{i}\left(u_{\tau}\right) \\
\hat{y}(t)= & \hat{\theta}_{1} \hat{x}_{1}(t)+\hat{\theta}_{n+1} \hat{x}_{2}(t) \cdots+\hat{\theta}_{n+m} \hat{x}_{m+1}(t), \\
& x_{i}^{L} \leq \hat{x}_{i}(t) \leq x_{i}^{U},(i=1,2, \mathrm{~L}, n), \\
& \theta_{i}^{L} \leq \hat{\theta}_{i} \leq \theta_{i}^{U},(i=1,2, \mathrm{~L}, n+m+K),
\end{aligned}\right.
$$

where $u_{\tau}(t)$ and $\mathrm{y}(t)$ are the input and output signal of the CT Hammerstein system; $x_{i}^{L}, x_{i}^{U}(i=$ $1,2, \cdots, n)$ are the lower bounds and upper bounds of the state variable $\hat{x}_{i}(t)$ and $\theta_{i}^{L}, \theta_{i}^{U}(i=$ $1,2, \cdots, n+m+K)$ are the lower bounds and upper bounds of the estimation parameters. The initial conditions of the states are set as

$$
\hat{x}_{1}\left(t_{0}\right)=\hat{x}_{2}\left(t_{0}\right)=\cdots=\hat{x}_{n}\left(t_{0}\right)=0 \text {. }
$$

The initial conditions can be considered as the boundary constraints of the optimal problem. Call back to (40), the parameter estimations of the CT Hammerstein system can be derived with

$$
\left\{\begin{array}{l}
\hat{a}_{1}=\frac{\hat{\theta}_{2}}{\hat{\theta}_{1}}, \hat{a}_{2}=\frac{\hat{\theta}_{3}}{\hat{\theta}_{1}}, \cdots, \hat{a}_{n-1}=\frac{\hat{\theta}_{n}}{\hat{\theta}_{1}}, \hat{a}_{n}=\frac{1}{\hat{\theta}_{1}}, \\
\hat{b}_{1}=\frac{\hat{\theta}_{n+1}}{\hat{\theta}_{1}}, \hat{b}_{2}=\frac{\hat{\theta}_{n+2}}{\hat{\theta}_{1}}, \cdots, \hat{b}_{m}=\frac{\hat{\theta}_{n+m}}{\hat{\theta}_{1}}, \\
\hat{\alpha}_{1}=\hat{\theta}_{n+m+1}, \hat{\alpha}_{2}=\hat{\theta}_{n+m+2}, \cdots, \hat{\alpha}_{K}=\hat{\theta}_{n+m+K} .
\end{array}\right.
$$

\section{Gauss pseudospectral discretization of CT Hammerstein system}

Let us transcribe the CT optimal problem for the Hammerstein parameters identification shown in (43)-(47) into a NLP problems by AGPM discussed in subsection 3.2. The number of LG points is equal to $N$, the $\mathrm{I} / \mathrm{O}$ data is measured from $t_{0}$ to $t_{f}$. Therefore, the discretized optimal problem can be obtained as the following description. 
Minimize the cost functional

$$
J=\frac{t_{f}-t_{0}}{2} \sum_{k=1}^{N} \omega_{k}\left(y\left(\tau_{k}\right)-\hat{y}\left(\tau_{k}\right)\right)^{2},
$$

subject to

$$
\left\{\begin{array}{c}
\sum_{l=0}^{N} D_{k l} \hat{x}_{1}\left(\tau_{l}\right)=\frac{t_{f}-t_{0}}{2} \hat{x}_{2}\left(\tau_{k}\right) \\
\sum_{l=0}^{N} D_{k l} \hat{x}_{2}\left(\tau_{l}\right)=\frac{t_{f}-t_{0}}{2} \hat{x}_{3}\left(\tau_{k}\right) \\
\vdots \\
\sum_{l=0}^{N} D_{k l} \hat{x}_{n}\left(\tau_{l}\right)=\frac{t_{f}-t_{0}}{2}\left(-\hat{\theta}_{1} \hat{x}_{1}\left(\tau_{k}\right)-\hat{\theta}_{2} \hat{x}_{2}\left(\tau_{k}\right) \cdots-\hat{\theta}_{n} \hat{x}_{n}\left(\tau_{k}\right)+\sum_{i=1}^{K} \hat{\theta}_{n+m+i} \phi_{i}\left(u_{\tau}\right)\right) \\
\hat{y}\left(\tau_{k}\right)=\hat{\theta}_{1} \hat{x}_{1}\left(\tau_{k}\right)+\hat{\theta}_{n+1} \hat{x}_{2}\left(\tau_{k}\right) \cdots+\hat{\theta}_{n+m} \hat{x}_{m+1}\left(\tau_{k}\right) \\
\hat{x}_{i}\left(\tau_{N+1}\right)=\hat{x}_{i}\left(\tau_{0}\right)+\sum_{k=1}^{N} \sum_{l=0}^{N} \omega_{k} D_{k l} \hat{x}_{i}\left(\tau_{l}\right),(i=1, \cdots, n), \\
\hat{x}_{1}\left(\tau_{0}\right)=\hat{x}_{2}\left(\tau_{0}\right)=\cdots=\hat{x}_{n}\left(\tau_{0}\right)=0 . \\
x_{i}^{L} \leq \hat{x}_{i}\left(\tau_{k}\right) \leq x_{i}^{U},(i=1,2, \mathrm{~L}, n ; k=0,1, \mathrm{~L}, N+1), \\
\theta_{i}^{L} \leq \hat{\theta}_{i} \leq \theta_{i}^{U},(i=1,2, \mathrm{~L}, n+m+K) .
\end{array}\right.
$$

NLP problem, described in (49)-(54), could be solved by Sequential Quadratic Programming (SQP) methods.In this paper, we use the KNITRO solver and MATLAB implementation environment to solve the transcribed NLP problem from the CT Hammerstein system.

\subsection{Illustrative Examples}

The simulation examples proposed in the paper to illustrate the AGPM are cited in Ref.[16]. The CT Hammerstein system is considered as a fourth-order nonlinear function with a second degree dynamic linear system. The CT Hammerstein system is expressed as

$$
\left\{\begin{array}{l}
\omega(t)=u(t)+0.628 u^{2}(t)+0.123 u^{3}(t)-0.036 u^{4}(t), \\
G(s)=\frac{1}{0.125 s^{2}+0.5 s+1} .
\end{array}\right.
$$

Set up $K=5$ as the number for Lagrange polynomials to approximate the static nonlinearity of the Hammerstein system. Then the Hammerstein system is identified by increasing the number of LG points with 30, 40, 50, respectively. In Figs.3. The black line represents the true system, and the blue dashed line, the red dotted line and the green dash-dot line represent the estimation results of AGPM algorithm with 30, 40, 50 LG nodes respectively. Fig.3.a show the nonlinearity part of the CT Hammerstein examples with the input range of $[-5,5]$. Fig.3.b display the bode diagram of the linear dynamic system. It is obvious that the estimated parameters can be well-matched with the true system using less than 50 interpolated LG nodes, and the more nodes we use, the more accurate for the estimation. It means that the AGPM can quickly converge to the true values and can decrease the computation time for the identification task. 


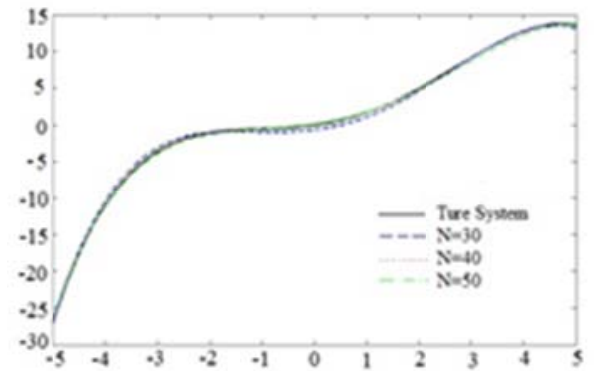

a) The plot of the identified static nonlinearity

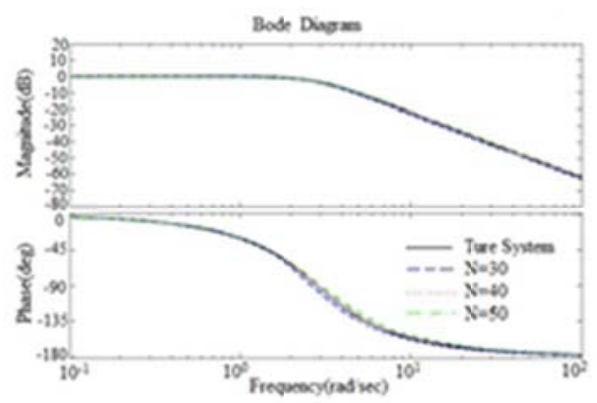

b) The Bode plot of the identified linear system

Fig. 3 The plot of the identified Hammerstein system for the example

\section{Conclusion}

In this paper, AGPM is proposed to identify the parameters of the CT Hammerstein system. In order to properly apply AGPM for the identification problem, it is necessary to make some transformation for the original CT Hammerstein system, including the approximation for the nonlinearity with Lagrangian polynomials and the state-space expression of the linear part. A minimization optimization to the output error of the CT Hammerstein system is constructed for the parameters identification. AGPM is applied to discretize the CT minimal optimization into a NLP problem. AGPM uses a linear constraint for the endpoint state variables instead of a nonlinear one in the general GPM, which can speed up the computation of the identification, then the NLP problem is solved with the well-developed SQP method. As a result, AGPM derives good-performance results with few LG interpolated nodes for two typical numerical examples. In the future work, we would like to extend AGPM to the CT Wiener model, and other nonlinear systems, and also hopefully to do the further research on the colored noise.

\section{Acknowledgments}

This work was financially supported by "the General Armaments fund (9140C3007081005)" "the School independent special (2010XQTR05)".

\section{References}

[1] Zhao Wen-Xiao and Chen Han-Fu. Adaptive Tracking and Recursive Identification for Hammerstein Systems [J]. Automatica, 2009, 45(12): 2773-2783.

[2] Chen Han-Fu. Pathwise convergence of recursive identification algorithms for Hammerstein systems [J]. IEEE Transactions on Automatic Control, 2004, 49(10): 1641-1649.

[3] $\mathrm{Mu}$ Bi-Qiang and Chen Han-Fu. Recursive Identification of MIMO Wiener Systems [J]. IEEE Transactions on Automatic Control, 2013, 58(3): 802-808.

[4] Ni Boyi, Garnier Hugues, and Gilson Marion. A Refined Instrumentall Variable Method for Hammerstein-Wiener Continuous-Time Model Identification [J]. System Identification, 2012, 16(1): 1061-1066.

[5] Han Younghee. Block-oriented Nonlinear System Identification Using Semidefinite Programming [D]. University of California, San Diego, 2012.

[6] Ding F. and Chen Tongwen. Identification of Hammerstein nonlinear ARMAX systems [J]. Automatica, 2005, 41(9): 1479-1489. 
[7] Ding F., Liu X. P., and Liu G. Identification methods for Hammerstein nonlinear systems [J]. Digital Signal Processing, 2011, 21(2): 215-238.

[8] Bai Er-Wei. An optimal two-stage identification algorithm for Hammerstein-Wiener nonlinear systems [J]. Lecture Notes in Control and Information Sciences, 2010, 404(27-34.

[9] Vanbeylen L., Pintelon R., and Schoukens J. Blind maximum likelihood identification of Hammerstein systems [J]. Automatica, 2008, 44(12): 3139-3146.

[10]Bai E. W. Frequency domain identification of Hammerstein models [J]. IEEE Transactions on Automatic Control, 2003, 48(4): 530-542.

[11]Dai Ming-Xiang, Zhang Jing-Xin, and Chai Li. Nuclear norm minimization in subspace based continuous-time Hammerstein system identification [C]. in 11th IEEE International Conference on Control \& Automation (ICCA), 2014, pp. 1269-1274.

[12] Greblicki W. Continuous-time Hammerstein system identification [J]. IEEE Transactions on Automatic Control, 2000, 45(6): 1232-1236.

[13]Greblicki W. Continuous-time Hammerstein system identification from sampled data [J]. IEEE Transactions on Automatic Control, 2006, 51(7): 1195-1200.

[14]Cheng Xi-Ming., Hu Xiao-Song., and Sun Feng-Chun. Parameter identification of continuous time Hammerstein system from step responses [C]. in 4th IEEE Conference on Industrial Electronics and Applications, 2009, pp. 3005-3010.

[15]Sun Yong, Zhang Mao-Rui, and Li He. New costate estimation of Gauss pseudospectral method for nonlinear optimal control problem [C]. in International Workshop on Engineering Application Research, Hangzhou, China, 2011, pp. 31-36.

[16]Benson David. A Gauss pseudospectral transcription for optimal control [D]. Massachusetts Institute of Technology, 2004. 